\title{
Pt(111)-Alloy Surfaces for Non-Activated OOH Dissociation
}

\author{
Wahyu Tri Cahyanto \\ Department of Precision Science and Technology and Applied Physics, \\ Graduate School of Engineering, Osaka University, Suita, Osaka 565-0871, Japan and \\ Department of Physics, Faculty of Science and Engineering, UNSOED, \\ Jl. Dr. Soeparno, Karangwangkal, Purwokerto 53123, Indonesia \\ Mary Clare Escaño and Hideaki Kasai* \\ Department of Precision Science and Technology and Applied Physics, \\ Graduate School of Engineering, Osaka University, Suita, Osaka 565-0871, Japan \\ Ryan Lacdao Arevalo \\ Department of Precision Science and Technology and Applied Physics, \\ Graduate School of Engineering, Osaka University, Suita, Osaka 565-0871, Japan and \\ 3Department of Physical Sciences, Philippine Normal University, Manila 1000, Philippines \\ (Received 10 June 2011; Accepted 26 August 2011; Published 27 September 2011)
}

\begin{abstract}
We present a density functional theory calculation for the adsorption and dissociation of $\mathrm{OOH}$ on $\mathrm{Pt}(111)$ and $\mathrm{Pt}(111)$-alloy surfaces. We confirmed the theoretical understanding of an activated $\mathrm{OOH}$ dissociation on $\mathrm{Pt}(111)$ surface and on small Pt clusters. Interestingly, in this work, we found an existence of a "barrierless" $\mathrm{OOH}$ dissociation on several Pt-binary and ternary alloy surfaces with $\mathrm{Ru}$ and Mo as alloying components: $\mathrm{PtRu}$ and PtRuMo. Here, we demonstrate how such reaction proceeds and discuss the role of $\mathrm{Ru}-\mathrm{O}$ and $\mathrm{Mo}-\mathrm{O}$ in the spontaneous $\mathrm{OOH}$ dissociation in these systems. The reaction energetics of $\mathrm{OOH}$ specie is one of the most sought fundamental surface science studies due to its importance in many catalytic and surface reactions such as hydrogen fuel cell. [DOI: 10.1380/ejssnt.2011.352]
\end{abstract}

Keywords: Density functional theory; Oxygen adsorption; OOH dissociation; Platinum; Ruthenium-based binary alloy; Molybdenum-based ternary alloys

\section{INTRODUCTION}

Among the many catalytic and surface reaction processes related to fuel cell development, the adsorption and dissociation of $\mathrm{OOH}$ species are considered one of the most important elementary steps. For instance, $\mathrm{OOH}_{\mathrm{ad}}$ is the main product of the first step in $\mathrm{O}_{2}$ reduction on metal surfaces [1-3]. It has been observed that the protonation of $\mathrm{O}_{2}$ comes before $\mathrm{O}-\mathrm{O}$ bond scission, revealing the utmost importance of $\mathrm{OOH}$ dissociation. The energetics of the dissociated components $\left(\mathrm{O}_{\mathrm{ad}}\right.$ and $\left.\mathrm{OH}_{\mathrm{ad}}\right)$ also significantly influence the over-all reaction as it dictates subsequent reaction steps such as protonation [3, 4] and oxidation of other surface species such as CO $[5,6]$. However, most of the fundamental surface science studies are devoted to the understanding of $\mathrm{O}$ and $\mathrm{OH}$ adsorption on metal or alloy catalysts [7-10], which are generally much simpler to treat. The challenge posed by a more complex $\mathrm{OOH}$ adsorption hinders both electrochemical and surface science studies. So far, the $\mathrm{OOH}$ adsorption and dissociation is understood on $\mathrm{Pt}(111)$ [11] and small $\mathrm{Pt}$ clusters $[12,13]$, where there is a general consensus that the $\mathrm{OOH}$ dissociation into $\mathrm{O}_{\mathrm{ad}}$ and $\mathrm{OH}_{\mathrm{ad}}$ components is activated. $\mathrm{OOH}$ adsorption is also studied in various $\mathrm{Pt}$ transition metal alloys and sandwich structures $[14,16]$. In this paper, we first report the non-activated dissociation of $\mathrm{OOH}$ on binary and ternary metal alloy systems: PtRu and PtRuMo. By comparison of the reactivity of each of the surface metal alloys and the clean $\operatorname{Pt}(111)$ system, we discuss the origin of such reaction.

*Corresponding author: kasai@dyn.ap.eng.osaka-u.ac.jp

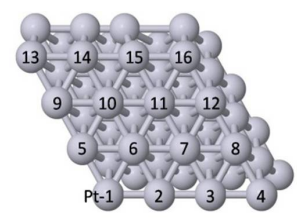

(a)

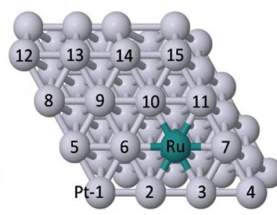

(b)

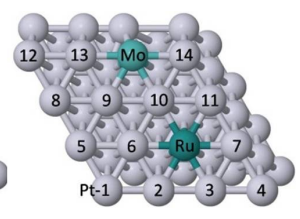

(c)
FIG. 1: (a) Pure Pt surface, (b) PtRu, and (c) PtRuMo. The $\mathrm{Pt}$ atoms are numbered and the Mo and Ru locations are depicted in both (b) and (c) accordingly.

\section{COMPUTATIONAL METHOD}

The theoretical study is based on total energy calculations within the density functional theory (DFT) as implemented in Vienna ab initio simulation package (VASP) [17]. The interaction between ions and electrons is described using projector augmented wave (PAW) method [18, 19]. A plane wave basis set with energy cut-off of $400 \mathrm{eV}$ is used. For the exchange-correlation functional, we employed the generalized gradient approximation (GGA) based on Perdew-Burke-Ernzerhof (PBE) [20, 21]. As shown in Fig. 1, we considered three model metal surfaces: Pt, PtRu and PtRuMo. PtRu and PtRuMo are denoted as surface metal alloys. The surface atomic ratio mimics the same metal alloy atomic ratio in experiments [22, 23]. Also, theoretically, it conforms to the differences in the segregation energies of $\mathrm{Pt}, \mathrm{Ru}$ and Mo [24], that is, positive surface segregation energy of the alloying components indicates a considerable number of host metal atom $(\mathrm{Pt})$ on the surface. These fcc(111) metal surfaces are all modeled using supercell approach where 


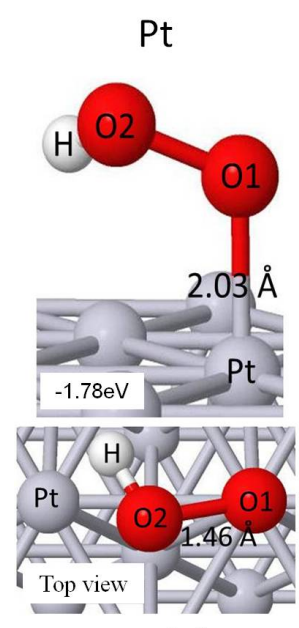

(a)

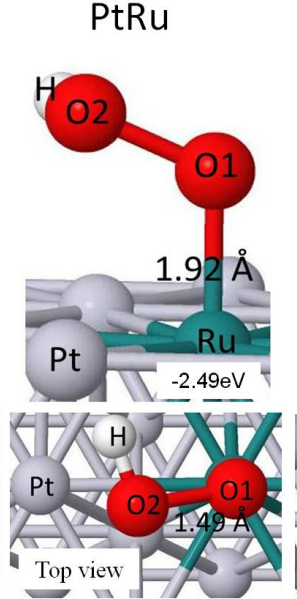

(b)
PtRuMo

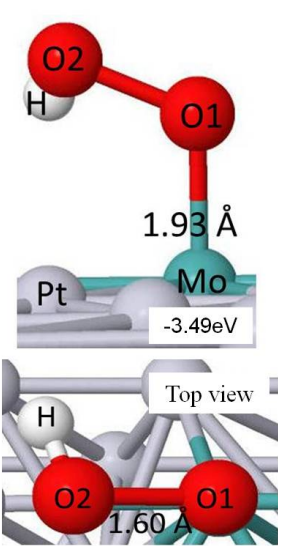

(c)
FIG. 2: $\mathrm{OOH}_{\mathrm{ad}}$ on (a) pure Pt surface; (b) PtRu; (c) PtRuMo. Top and bottom panels correspond to side and top views of the adsorbate/substrate system. The metal-O1 and O1-O2 bond lengths and the adsorption energies are indicated.

the slab is composed of three atomic layers in a $(4 \times 4)$ unit cell. Because of the periodic boundary conditions imposed via the supercell, a $\sim 15.0 \AA$ of vacuum along $z$ axis is introduced to avoid interaction between surfaces. The surface Brillouin zone integrations were performed on a grid of $(5 \times 5 \times 1)$ Monkhorst-Pack k-points [25] using Methfessel-Paxton smearing [26] of $\sigma=0.2 \mathrm{eV}$. For the adsorption calculations, all the atomic positions are allowed to relax except for the bottom layer, which is constrained to its bulk position. Since adsorption is made on one side of the slab, a dipole correction is introduced. The size of the unit cell leads to an adsorption coverage of $\sim 1 / 16 \mathrm{ML}$ with respect to the number of surface atoms. Convergence of numerical results with respect to the slab thickness, the kinetic energy cut-off and the k-point set is established.

\section{RESULTS AND DISCUSSION}

\section{A. Initial $\mathrm{OOH}$ adsorption}

The most stable $\mathrm{OOH}$ adsorption configuration on the surfaces is exhaustively explored by a potential energy scan on all major sites (top, fcc-hollow, hcp-hollow and bridge) using various $\mathrm{OOH}$ conformations with the surface. The potential energy $\left(E_{\text {ads }}\right)$ is calculated as: $E_{\text {ads }}=E_{\text {system }}-\left(E_{\text {slab }}+E_{\text {gas-phase adsorbate }}\right)$ where $E_{\text {system }}, E_{\text {slab }}$, and $E_{\text {gas-pahse adsorbate }}$ correspond to the total energy of the adsorbate/substrate system, bare slab, and isolated molecule, respectively. The adsorption on $\mathrm{OOH}$ on $\mathrm{Pt}(111)$, in this work, prefers an O-down configuration over Pt top site (see Fig. 2) with the $\mathrm{O}-\mathrm{O}$ bond tilted up (i.e. end-on adsorption). This structure is in excellent agreement with other DFT results [11]. Because of finite size effects, the adsorption energy slightly differs from that of very small $\mathrm{Pt}$ clusters, albeit within the range of those in bigger Pt-cluster sizes $[12,13]$. Interestingly, the end-on adsorption with the O-end of $\mathrm{OOH}_{\mathrm{ad}}$ (here labeled as O1) is also observed for PtRu and PtRuMo surfaces, but the metal-O1 bond is exclusive to the alloying component. That is, $\mathrm{Ru}-\mathrm{O} 1$ bond is formed in $\mathrm{PtRu}$ while Mo-O1 is formed in PtRuMo. This confirms the "capturing" of $\mathrm{O} 1$ by the transition metal atom, X (X: $\mathrm{Ni}, \mathrm{Co}, \mathrm{Cr}$ ), which is substituted in a Pt cluster with an atomic ratio $(\mathrm{Pt}: \mathrm{X})$ of $2: 1$ [14]. We note that in $\mathrm{PtRu}$ and PtRuMo, the $\mathrm{OOH}_{\mathrm{ad}}$ is further stabilized, as verified by the shortened metal-O1 bond lengths. Moreover, the $\mathrm{O} 1-\mathrm{O} 2$ (O2 is the oxygen atom attached to $\mathrm{H})$ bond length is already significantly stretched in Pt-surface alloys as shown in Fig. 2, top views. Specifically, the O1-O2 bond length in PtRu is $\sim 1.49 \AA$. This is a $\sim 0.03 \AA$ stretch in $\mathrm{O} 1-\mathrm{O} 2$ bond with respect to that of $\mathrm{Pt}(1.46 \AA)$. For PtRuMo, the $\mathrm{O}-\mathrm{O}$ bond is elongated by $\sim 0.11 \AA$ with respect to $\mathrm{PtRu}$ and $\sim 0.14 \AA$ with respect to $\mathrm{Pt}$. The role of this elongation at IS in $\mathrm{OOH}$ dissociation will be discussed further later.

\section{B. Dissociation of $\mathrm{OOH}$}

Next, we calculated the energies of the transition state (TS) and the final state (FS) of $\mathrm{OOH}_{\mathrm{ad}}$ dissociation into $\mathrm{O}_{\mathrm{ads}}+\mathrm{OH}_{\mathrm{ads}}$ on Pt, PtRu, and PtRuMo surfaces. The transition state is located by determining the saddle point in the minimum energy path towards the final configuration (FS) via potential energy surface (PES) scan. The saddle point is the maximum of the minimum energy path. The exploration of potential minimum involves calculating the potential energy $(\mathrm{PE})$ as a function of the coordinates of the molecule, which represent its degrees of freedom. The variation of coordinates involves changing: (1) the $\mathrm{O}-\mathrm{OH}$ bond lengths $(\Delta r) ;(2) \mathrm{OOH}$ distance from the surface $(z)$; and $(3) \mathrm{OOH}$ distance from adsorbed state to dissociated state $(x, y)$, with an increment of $0.17 \AA$. From the calculated $\mathrm{PE}$ values, the minimum $\mathrm{PE}$ is traced and the point of maximum energy is the TS. Since the location of TS is important for mechanistic study of dissociation, we further checked the TS by displacing the molecule at this point in all possible directions using smaller increments $(0.1 \AA)$. Such displacements did not give higher PE. The energies of the three important points in the reaction path, i.e. initial state (IS), transition state (TS) and final state (FS), for $\mathrm{Pt}(111)$ is shown in black line in Fig. 3, left panel. Indeed, the dissociation of $\mathrm{OOH}$ is activated on $\mathrm{Pt}$. The calculated activation barrier $(0.39 \mathrm{eV})$ for $\mathrm{Pt}(111)$ is in good agreement with experiments $(0.44 \mathrm{eV}[27])$ and other DFT results $(0.25 \mathrm{eV}-0.56 \mathrm{eV}$ [11-13]). However, as shown in the reaction energy diagram for $\mathrm{PtRu}$ and PtRuMo, the presence of $\mathrm{Ru}$ and Mo on Pt surface remarkably eliminates the barrier. The reaction paths for these metal alloy systems are now downhill from zero PE at certain reaction coordinates. The TS in these flat reaction paths is defined as the starting point for a downhill path. Thus, we show a non-activated dissociation phenomenon for $\mathrm{OOH}$ species on $\mathrm{Pt}$ metal surfaces in the form of binary and ternary alloys using $\mathrm{Ru}$ and Mo as alloying components. 


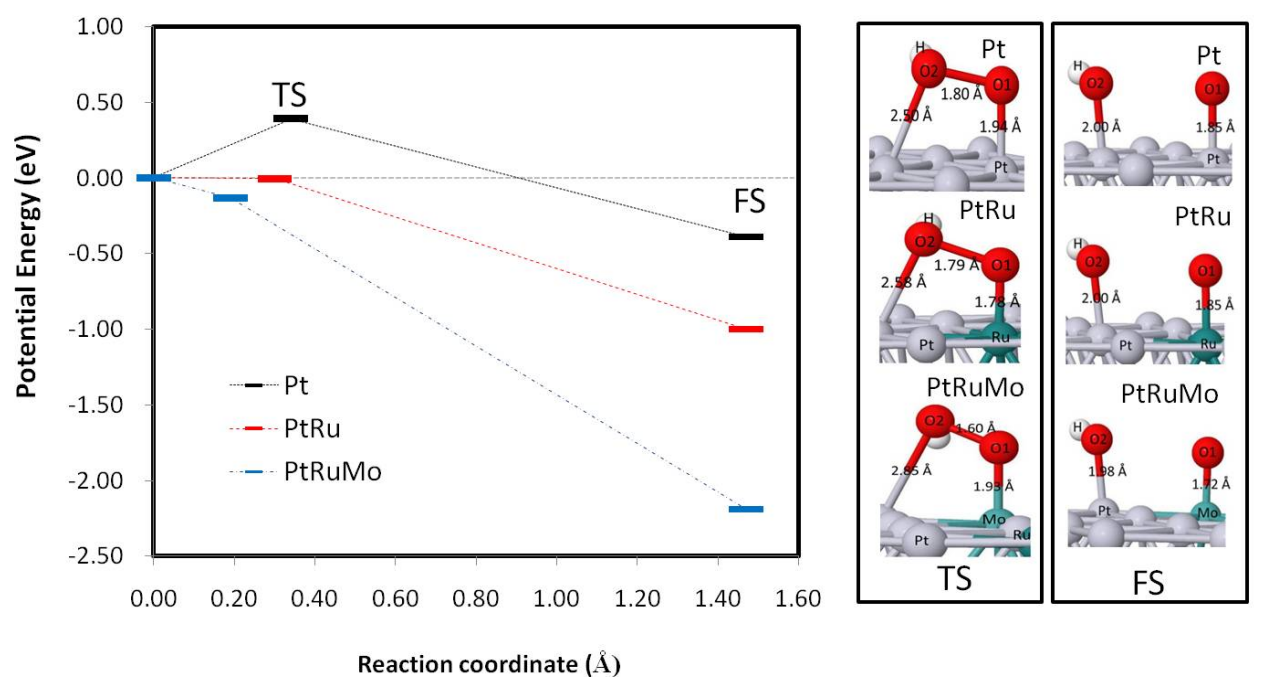

FIG. 3: Reaction diagram for $\mathrm{OOH}_{\mathrm{ads}}$ into $\mathrm{O}_{\mathrm{ads}}+\mathrm{OH}_{\mathrm{ads}}$ on $\mathrm{Pt}, \mathrm{PtRu}$, and $\mathrm{PtRuMo}$. The corresponding transition and final states structures are given on the right. The change in the $\mathrm{O}-\mathrm{OH}$ bond with respect to IS, is shorter for PtRuMo since $\mathrm{O}-\mathrm{OH}$ at IS, is already significantly elongated in this system. Since, at PtRuMo, the reaction energy is immediately downhill after the IS, then the defined "TS" is the same as the IS. For, PtRu, the downhill path starts at reaction coordinate $\sim 0.40 \AA$.

\section{Dissociation mechanism}

Herein, we discuss the details and mechanism of $\mathrm{OOH}$ adsorption and dissociation on the model surfaces. First, we note that the trend in the relative stability of $\mathrm{OOH}_{\mathrm{ad}}$ among the metal surfaces at TS and FS is maintained, that is, with PtRuMo giving the strongest binding, followed by PtRu and then Pt. A careful inspection of the TS and FS of all the surfaces, especially that which involves metal- $\mathrm{O} 2 \mathrm{H}$ and metal-O 1 bonds, reveals that the metal-OOH interaction is only governed by how much the oxygen (O1) is bound to the surface. At the TS, the metal-O1 bond length decreases in the following order: $\mathrm{Pt}>\mathrm{PtRu}>\mathrm{PtRuMo}$ which suggests that the strength of metal-OOH interaction at TS follows the same order. We note that this metal-O1 bond is the same bond that holds $\mathrm{OOH}$ at IS in an end-on configuration, as discussed previously. We recall that, at IS, the O1-O2 bond is already well-loosened. Hence, such IS configurations on Pt-surface alloys seems to contribute to the enhancement of $\mathrm{OOH}$ dissociation. The same trend is observed by Balbuena et al. [13], wherein the $\mathrm{O}-\mathrm{O}$ bond elongation in the initial $\mathrm{OOH}$ adsorption (IS), although very small $(\sim 0.01 \AA)$ on pure Pt cluster, correlates well with the decrease the activation barrier for $\mathrm{OOH}$ dissociation.

In the following, we show how IS and the TS are correlated. First, we compared the TS and IS for $\mathrm{Pt}$ and PtRu. In Table I, we show the charge gained by O1, O2 and $\mathrm{H}$ atoms at $\mathrm{TS}$ with respect to that of clean $\mathrm{Pt}$ using Bader charge analysis [28]. We note that at TS, the O1 atom in $\mathrm{PtRu}$, gains $0.126 e^{-}, \mathrm{O} 2$ atom gains $0.052 e^{-}$ and the $\mathrm{H}$ atom gains $0.0 e^{-}$with respect to $\mathrm{Pt}$. It is clear that the gain in charge is much more significant in $\mathrm{O} 1$ than in $\mathrm{O} 2$ and $\mathrm{H}$. To compare PtRu and PtRuMo, we calculated the same gain in charge for each atom as described above. With respect to PtRu at TS, O1 gained more charge $\left(0.18 e^{-}\right)$than that of PtRu with respect to $\mathrm{Pt}$. However, for the case of $\mathrm{O} 2$, the gain in charge is lesser. The presence of Mo (PtRu vs PtRuMo), therefore, increases the net charge in $\mathrm{O} 1$ atom but not in $\mathrm{O} 2$ and $\mathrm{H}$. The presence of $\mathrm{Ru}(\mathrm{Pt}$ vs $\mathrm{PtRu}$ ) increases the net charge in $\mathrm{O} 1$ atom and minimally on $\mathrm{O} 2$. In all cases, the charge gained by $\mathrm{O} 1$ is large whether on PtRu or PtRuMo. Taking all the cases above, we note that IS and TS are correlated via metal-O1 bonding (i.e. $\mathrm{Pt}-\mathrm{O} 1, \mathrm{Ru}-\mathrm{O} 1$ and $\mathrm{Mo}-\mathrm{O} 1)$. The TS is governed by a large net charge gain on $\mathrm{O} 1$, while the IS is governed by significant $\mathrm{O}-\mathrm{O}$ bond elongation by an end-on $\mathrm{OOH}$ adsorption via $\mathrm{O} 1$. We note that there is very little difference in the charge density at Mo for PtRu and PtRuMo, hence, the Ru does significantly affect the Mo reactivity to O1.

The next question therefore arises as to how the IS and TS characteristics above affect the energetics of $\mathrm{OOH}$ dissociation. First, we note that as $\mathrm{OOH}$ adsorbs on the metal surface, the adsorbate orbital splits into bonding and anti-bonding states upon interaction with metal $d$ orbital. It is however in the filling of the anti-bonding states that dictates whether a barrier stands in the way or not, since such occupation results to repulsive $\mathrm{O}-\mathrm{O}$ interaction. So far there have been no studies on intramolecular charge transfer effects in $\mathrm{OOH}$ and therefore, charge transfer between metal and adsorbate is likely. In $\mathrm{PtRu}$, the difference in the charge transferred to the adsorbate at TS with respect to $\mathrm{Pt}$ is $0.18 e^{-}(\mathrm{O} 1+\mathrm{O} 2+\mathrm{H}$ charges in Table I). For PtRuMo, the charge transfer difference is much larger, $0.34 e^{-}$. For both cases, the $\mathrm{OOH}$ dissociation is barrierless. This suggests that, at least, an increase of $0.18 e^{-}$in terms of charge transfer to adsorbate must be achieved to eliminate the barrier in $\operatorname{Pt}(111)$.

Since the TS is related to IS via the $\mathrm{OOH}$ end-on adsorption by metal-O1 bonding, then, corollary to the charge transfer effects at TS described above, the initial $\mathrm{OOH}$ adsorption should lead to an $\mathrm{O} 1-\mathrm{O} 2$ bond length of at least $1.49 \AA$ to lead to an easy dissociation as observed in PtRu. To check whether these limits are reliable, we evaluate the existence of non-spontaneous $\mathrm{OOH}$ 
TABLE I: Charge gain $\left(e^{-}\right)$in $\mathrm{O} 1, \mathrm{O} 2$ and $\mathrm{H}$ with respect to that of $\mathrm{Pt}(111)$ and $\mathrm{O}-\mathrm{O}$ bond lengths at TS and IS for Pt-surface alloys.

\begin{tabular}{ccccccc}
\hline \hline No. & Surfaces & \multicolumn{2}{c}{ Charge gain $\left(e^{-}\right)$at TS with respect to Pt } & \multicolumn{2}{c}{ O-O bond at } & \multicolumn{2}{c}{ O-O bond at } \\
& & $\mathrm{O} 1$ & $\mathrm{O} 2$ & $\mathrm{H})$ & 1.80 & $\mathrm{IS}(\AA)$ \\
\hline 1 & $\mathrm{Pt}$ & - & - & - & 1.79 & 1.46 \\
2 & $\mathrm{PtRu}$ & 0.126 & 0.052 & 0.000 & 1.60 & 1.49 \\
3 & PtRuMo & 0.300 & 0.037 & 0.000 & 1.60 \\
\hline \hline
\end{tabular}

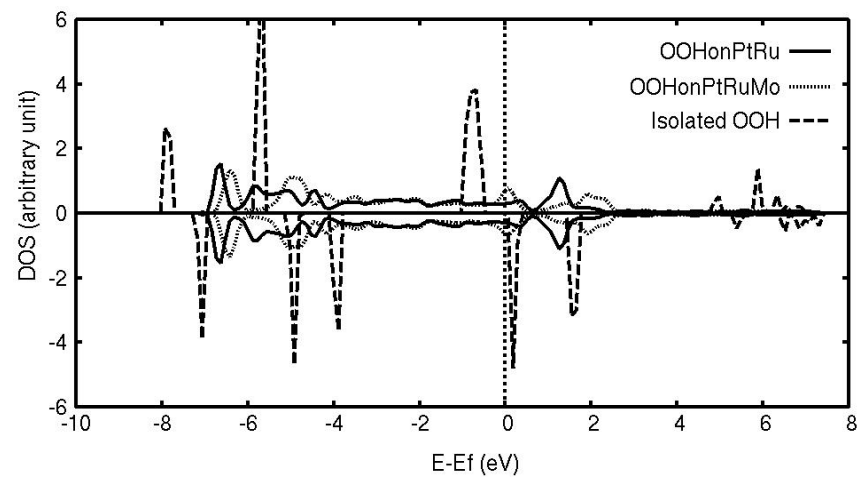

FIG. 4: Local density of states of isolated $\mathrm{OOH}$ and $\mathrm{OOH}$ at TS on PtRu and PtRuMo.

adsorption on Pt-alloys previously cited [14]. In all these clusters, the charge transfer to $\mathrm{OOH}$ at TS is well below $0.18 e^{-}$. Specifically, in $\mathrm{Pt}_{2} \mathrm{Ni}$, the charge transfer difference with respect to $\mathrm{Pt}$ cluster is $0.09 e^{-}$, almost the same as that in our $\mathrm{Pt}(111)$ calculation. The $\mathrm{O}-\mathrm{O}$ bond length at initial $\mathrm{OOH}$ adsorption in the $\mathrm{Pt}(111)$ is $1.457 \AA$ (or rounded off to 1.46 in Table I). This is the same as that of $\mathrm{Pt}_{2} \mathrm{Ni}$, which is $\sim 1.453 \AA$.

To verify this role of charge transferred to $\mathrm{OOH}$ on Pt-surface alloys further, we plotted the local density of states (LDOS) for isolated $\mathrm{OOH}$ and for $\mathrm{OOH}$ at TS on the Pt-surface alloys as shown in Fig. 4. We note that due to interaction with metal surface, the unoccupied spin down peak (anti-bonding state) right above $E_{\mathrm{F}}$ for isolated $\mathrm{OOH}$, disappears and the unoccupied peak higher in energy is broadened upon interaction with $\mathrm{PtRu}$ and PtRuMo. This verifies the occupation of the anti-bonding states via charge transfer from the metal. We also note that the broadening of the adsorbate states are more evident for PtRuMo. This charge transfer mechanism has long been correlated with $\mathrm{O}-\mathrm{O}$ elongation for many other oxygenated species [13, 29-31]. In particular, for $\mathrm{OOH}$ on $\mathrm{Pt}$ cluster, the relationship between increasing $\mathrm{Pt}-$ $\mathrm{Pt}$ distance and elongation of the $\mathrm{O}-\mathrm{O}$ bond is established [13]. Increasing the $\mathrm{Pt}-\mathrm{Pt}$ distance shifts the metal $d$-band closer to the $E_{\mathrm{F}}$ since the $\mathrm{Pt}-\mathrm{Pt}$ orbital overlap is less when the $\mathrm{Pt}-\mathrm{Pt}$ lateral distance is increased. If the Pt- $d$ band is close to $E_{\mathrm{F}}$, the density of states (DOS) at $E_{\mathrm{F}}$ increases, which in turn increases electron states available for charge transfer to the adsorbate.

Lastly, we discussed the importance of the reaction of $\mathrm{OOH}$ on metal catalyst. We note that dissociation of $\mathrm{OOH}$ with no energy cost is desired in some catalytic reactions. For instance, as mentioned in the introduction, studies on the presence of $\mathrm{OOH}$ in the oxidation reduction reaction (ORR) [32-34] and its selectivity towards water as end product on the cathode of hydrogen fuel cell, present the following elementary steps:

$$
\begin{aligned}
\mathrm{O}_{2}+e^{-}+\mathrm{H}^{+} & \rightarrow \mathrm{M}-\mathrm{OOH} \\
\mathrm{M}-\mathrm{OOH} & \rightarrow \mathrm{M}-\mathrm{O}+\mathrm{M}-\mathrm{OH} \\
\mathrm{M}-\mathrm{OH}+\mathrm{H}^{+} & \rightarrow \mathrm{M}-\mathrm{H}_{2} \mathrm{O}
\end{aligned}
$$

where $\mathrm{M}$ represents the metal catalyst. If (2) is slow, then a subsequent protonation would yield $\mathrm{M}-\mathrm{H}_{2} \mathrm{O}_{2}$ instead of $\mathrm{M}-\mathrm{H}_{2} \mathrm{O}$. This specie increases the overpotential on the cathode decreasing the effectiveness of fuel utilization. The presence of $\mathrm{H}_{2} \mathrm{O}_{2}$ also reduces the effectiveness of the catalyst, reducing the current density of the fuel cell. However, if $(1) \rightarrow(2)$ is spontaneous, then the chance of breaking of $\mathrm{OOH}$ into $\mathrm{O}_{\mathrm{ad}}$ and $\mathrm{OH}_{\mathrm{ad}}$ before the next protonation, can be enhanced, thus, minimizing the $\mathrm{H}_{2} \mathrm{O}_{2}$ pathway. Dissociation with least cost of energy is highly required for $\mathrm{OOH}$. In this work, we presented model Ptsurface alloys that can achieve such $\mathrm{OOH}$ energetics.

\section{CONCLUSION}

We conducted first principles calculation for the adsorption and dissociation of $\mathrm{OOH}$ on $\mathrm{Pt}$ and $\mathrm{Pt}$-surface alloys: PtRu and PtRuMo. We found that the adsorption of $\mathrm{OOH}$ is end-on on all systems. The dissociation of $\mathrm{OOH}_{\mathrm{ad}}$ into $\mathrm{O}_{\mathrm{ad}}$ and $\mathrm{OH}_{\mathrm{ad}}$ is activated on $\mathrm{Pt}(111)$ in agreement with experiments and other theoretical works. In this study, we introduce a non-activated dissociation of $\mathrm{OOH}$ over PtRu and PtRuMo. The dissociation is spontaneous due to the enhanced $\mathrm{Ru}-\mathrm{O} 1$ and $\mathrm{Mo}^{-} \mathrm{O} 1$ bonding (the $\mathrm{O}$ by which $\mathrm{OOH}$ initially forms bond with the surface). This bonding at IS and TS is governed by an enhanced charge transfer from the alloying component to the $\mathrm{OOH}$ via $\mathrm{O} 1$. We found that a charge transfer increase (with respect to $\mathrm{Pt}$ ) of $\sim 0.18 e^{-}$in $\mathrm{PtRu}$, eliminates the barrier observed in $\mathrm{Pt}(111)$. For PtRuMo, the charge transfer increase is much larger $\left(0.34 e^{-}\right)$. The importance of non-activated dissociation of $\mathrm{OOH}$ on metal-alloy systems is seen in the oxidation reduction reaction (ORR), which is often desired to be selective towards water formation. The very low cost to dissociate $\mathrm{OOH}$, which is an intermediate in the over-all ORR, prevents $\mathrm{H}_{2} \mathrm{O}_{2}$ formation and increases the chance for an $\mathrm{H}_{2} \mathrm{O}$ pathway. 


\section{Acknowledgments}

This work is supported by the Special Coordination Funds for the Global Center of Excellence (COE) program (H08) "Center of Excellence for Atomically Controlled Fabrication Technology" and Directorate General of Higher Education (DGHE), Department of National Education, Republic of Indonesia. M.C. Escaño would like to extend gratitude to Japan Society for Promotion of Science (JSPS) Young Researcher Fellowship for funding. R.L. Arevalo extends his gratitude to the Ministry of Education, Culture, Sports, Science and Technology (MEXT) for scholarship. W.T. Cahyanto would like to thank Dr. D.N. Son, Prof. E. Gyenge, Prof. H. Nakanishi and Mr. H. Kishi for fruitful discussions and technical support.
[1] N. M. Markovic, H. A. Gasteiger, and P. N. Ross Jr., J. Phys. Chem. 99, 3411 (1995).

[2] R. A. Sidik and A. B. Anderson, J. Electroanal. Chem. 528, 69 (2002).

[3] A. Udaykumar and M. Mavrikakis, Sur. Sci. 602, L89 (2008).

[4] J. K. Nørskov, J. Rossmeisl, A. Logadottir, L. Lindqvist, J. R. Kitchin, T. Bligaard, and H. Jonsson, J. Phys. Chem. B. 108, 17886 (2004).

[5] B. C. Han and G. Ceder, Phys. Rev. B 74, 205418 (2006).

[6] T. E. Shubina and M. T. M. Koper, Electrochemica Acta 47, 3621 (2002).

[7] J. Roques and A. B. Anderson, J. Electrochem. Soc. 151, E340 (2004).

[8] M. Wakisaka, H. Suzuki, S. Mitsui, H. Uchida, and M. Watanabe, Langmuir 25, 1897 (2009).

[9] A. Kokalj, A. Lesar, M. Hodošček, and M. Causà, J. Phys. Chem B 103, 7222 (1999).

[10] J. Roques and A. B. Anderson, J. Electrochem. Soc. 151, E85 (2004).

[11] M. P. Hyman and J. W. Medlin, J. Phys. Chem. B 110, 15338 (2006).

[12] Y. Wang and P. B. Balbuena, J. Chem. Theory Comput. 1, 935 (2005).

[13] Y. Ma and P.B. Balbuena, Chem. Phys. Lett. 447, 289 (2007).

[14] J. M. Seminario, L. A. Agapito, L. Yan, and P. B. Balbuena, Chem. Phys. Lett. 410, 275 (2005).

[15] D. C. Papageorgopoulos, M. Keijzer, and F. A. de Brujin, Electrochim. Acta 48, 197 (2002).

[16] M. P. Hyman and J. W. Medlin. J. Phys. Chem. C 111, 17052 (2007).

[17] G. Kresse and J. Furthmuller, Phys. Rev. B 54, 11169
(1996).

[18] G. Kresse and J. Joubert, Phys. Rev. B 59, 1758 (1999).

[19] P. E. Blochl, Phys. Rev. B50, 17953 (1994).

[20] J. P. Perdew, K. Burke, and M. Ernzerhof, Phys. Rev. Lett. 77, 1396 (1997).

[21] J. P. Perdew, K. Burke, and M. Ernzerhof, Phys. Rev. Lett. 78, 1396 (1997).

[22] K. R. Lee, M. K. Jeon, and S. I. Woo, Appl. Catal. B: Environ. 91, 428 (2009).

[23] Z. Wang, P. Zuo, and G. Yin, Fuel Cells 9, 106 (2009)

[24] A. V. Ruban, H. L. Skriver, and J. K. Nørskov, Phys. Rev. B 59, 15990 (1999).

[25] H. J. Monkhorst and J. D. Pack, Phys. Rev. B 13, 5188 (1976).

[26] M. Methfessel and A. Paxton, Phys. Rev. B 40, 3616 (1989)

[27] B. N. Grgur, N. M. Markovic, and P. N. Ross, Can. J. Chem. 75, 1465 (1997).

[28] R. Bader, Atoms in Molecules: A Quantum Theory (Oxford University Press, New York, 1990).

[29] S. R. Calvo and P. B. Balbuena, Surf. Sci. 601, 4786 (2007).

[30] P. B. Balbuena, S. R. Calvo, E. J. Lamas, P. F. Salazar, and J. M. Seminario, J. Phys. Chem. B 110, 17452 (2006).

[31] S. R. Calvo and P. B. Balbuena, Surf. Sci. 601, 165 (2007).

[32] M. Tsuda and H. Kasai, J. Phys. Soc. Jpn. 76, 024801 (2007).

[33] D. N. Son, H. Nakanishi, M. Y. David, and H. Kasai, J. Phys. Soc. Jpn. 78, 114601 (2009).

[34] D. N. Son, B. T. Cong, and H. Kasai, J. Nanosci. Nanotechnol. 11, 2983 (2011). 